\title{
Insatisfação com a massa corporal e uso de suplementos alimentares em frequentadoras de academias de ginástica em Juiz de Fora - MG
}

Body weight dissatisfaction and the intake of dietary supplements among women who go to gym in Juiz de Fora - MG

Insatisfacción con la masa corporal y suplementos alimenticios entre las mujeres que van al gimnasios en Juiz de Fora - MG

\author{
Carlos Roberto Ramos da Rosa Junior', Jéssica Fernades Viana ${ }^{\mathrm{II}}$, Marina Rodrigues Vieira de \\ Souza ${ }^{\mathrm{III}}$, Maria Elisa Caputo Ferreira ${ }^{\mathrm{IV}}$
}

\begin{abstract}
Resumo
O objetivo deste estudo foi verificar a associação entre insatisfação com a massa corporal e o uso de suplementos alimentares em mulheres que frequentam academias de ginástica em Juiz de Fora, MG. Os dados foram coletados em sete estabelecimentos. A insatisfação com a massa corporal foi avaliada pela diferença entre a massa corporal considerada ideal para si mesma e a massa corporal auto referida. De acordo com os resultados, conclui-se que a maior parte das mulheres apresentou insatisfação com a massa corporal, assim como mais de um terço utilizou suplementos alimentares, porém essas variáveis não estavam correlacionadas.
\end{abstract}

Palavras-chave: Imagem Corporal; Mulheres; Suplementos Nutricionais; Academias de Ginástica

\begin{abstract}
The aim of this study was to verify the association between body weight dissatisfaction and the use of dietary supplements in women who attend gyms in Juiz de Fora, MG, Brazil. Data were collected in seven different establishments. Body mass dissatisfaction was evaluated by the difference between the body weight considered ideal for herself and self-report body mass. According to the results, it is concluded that most of the women showed body mass dissatisfaction, as well as more than one-third used dietary supplements, but these variables were not correlated.
\end{abstract}

Keywords: Body Image; Woman; Dietary Supplements; Fitness Centers

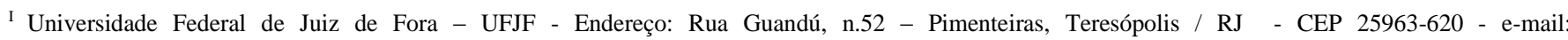
carlosramosjr.edfisica@gmail.com

II Universidade Federal de Juiz de Fora - UFJF - e-mail: jessicafviana91@ hotmail.com

III Universidade Federal de Juiz de Fora - UFJF - e-mail: mrvs_jf@ @otmail.com

${ }^{\text {IV }}$ Universidade Federal de Juiz de Fora - UFJF - e-mail: caputoferreira@terra.com.br
} 


\section{Resumen}

El objetivo de este estudio fue verificar la asociación entre insatisfacción con la masa corporal y el uso de alimentos suplementos en mujeres que asisten a gimnasios en Juiz de Fora, MG, Brasil. Los datos fueron recogidos en siete establecimientos diferentes. La insatisfacción con la masa corporal fue evaluada por la diferencia entre el peso corporal considerado ideal por sí misma y peso corporal auto referido. Según los resultados, se concluye que la mayoría de las mujeres mostró insatisfacción con la masa corporal, así como más de un tercio usaba suplementos alimenticios, pero estas variables no se correlacionaron.

Palabras clave: Imagen Corporal; Mujeres; Suplementos Dietéticos; Gimnansios

\section{Introdução}

Na sociedade contemporânea, o desejo pela construção de um corpo ideal tornou-se o principal objetivo, a maioria das vezes, esse anseio pode ser estimulado por fatores socioculturais tais como mídias, familiares e amigos (AMARAL; CARVALHO; FERREIRA, 2014; THORNSBORROW, T. et al. 2020). Segundo Iriart, Chaves, Orleans (2009), a exacerbada valorização da aparência física apresenta-se como um processo no qual o corpo do próprio indivíduo assume um papel fundamental na manifestação dos anseios pessoais e na construção da própria identidade.

Observa-se a divulgação e difusão de diferentes modelos corporais entre as variadas culturas ao redor do mundo, levando os indivíduos a buscarem padrões irreais de aparência física (THORNBORROW et al., 2020). É nesse sentido que a insatisfação corporal talvez seja um dos principais motivos que leva as pessoas a se engajarem em programas de atividades físicas (DAMASCENO et al., 2005).

A imagem corporal pode ser entendida como a maneira pela qual o indivíduo percebe e se sente em relação ao próprio corpo (CASH; SMOLAK, 2011).A dimensão atitudinal desse constructo engloba a insatisfação que se refere a avaliações negativas que o indivíduo possui sobre a aparência, e que pode estar relacionada com distorções na forma como se vê e representafisicamente (GROGAN, 2008).Diferentes aspectos da insatisfação corporal têm-se apresentado como foco de variadas investigações avaliando esse constructo em indivíduos adultos (CARVALHO; CASTRO; FERREIRA, 2014).

Os estudos sobre a imagem corporal de mulheres brasileiras sugerem a existência de insatisfação com a aparência em estudantes adolescentes (ADAMI et al., 2008), universitárias (ALVARENGA et al, 2010; KAKESHITA; ALMEIDA, 2006) e idosas fisicamente ativas (PEREIRA et al., 2009). De forma especial, também se observa esse descontentamento em mulheres praticantes de atividades físicas (DAMASCENO et al., 2005; ROSSI; TIRAPEGUI, 2018). 
A contínua busca por uma aparência física socialmente idealizada parece gerar elevados níveis de insatisfação corporal nos indivíduos, o que pode também estimular o uso de diferentes tipos de substâncias - suplementos alimentares e esteroides anabólicos -, numa tentativa de aumentar a satisfação com o próprio corpo (HILDEBRANT; LAI, 2011; SCHULER; ROCHA, 2018).

Segundo o Conselho Federal de Nutrição - CFN (2005), os suplementos alimentares servem para complementar, com calorias e ou nutrientes a dieta diária de uma pessoa saudável, em casos em que sua ingestão, a partir da alimentação, seja insuficiente. Contudo, essas substâncias não deveriam substituir os alimentos e nem serem considerados uma fonte alimentar exclusiva.

A respeito do aumento no consumo de suplementos alimentares nos dias atuais, Linhares e Lima (2006) destacam que isso possivelmente ocorre devido ao fato de serem considerados recursos para suprir qualquer tipo de deficiência que o organismo apresente. Já, Silva, Ferreira Júnior e Neves (2012) sugerem que esse quadro também pode ser reflexo do aumento no número de novos suplementos alimentares no mercado.

Apesar do consumo de suplementos alimentares parece ser menor em mulheres quando comparado aos homens (SILVA; FERREIRA JÚNIOR; NEVES, 2012; SOUZA; CENI, 2014), a busca por uma aparência física idealizada socialmente tem feito com que um número cada vez maior de mulheres faça uso desse tipo de substância (ALVES et al. 2017; MOREIRA; NAVARRO; NAVARRO, 2014; SCHULER; ROCHA, 2018).

Diante desse panorama, o desenvolvimento deste estudo tornou-se importante no intuito de elucidar possíveis relações entre as mulheres frequentadoras de academias de ginástica, a insatisfação com a massa corporal e o uso de suplementos alimentares, no sentido de fornecer informações que sirvam para subsidiar as intervenções de profissionais de Educação Física e Nutricionistas junto a essa população. Sendo assim, o objetivo deste estudo foi verificar a associação entre a insatisfação com a massa corporal e o uso de suplementos alimentares em mulheres que frequentam academias de ginástica na cidade de Juiz de Fora, MG.

\section{Metodologia}

\subsection{Caracterização do Estudo e Aspectos Éticos}

Este estudo pode ser considerado uma pesquisa quantitativa de corte transversal, descritiva e correlacional (THOMAS; NELSON; SILVERMAN, 2012), tendo seu projeto aprovado pelo Comitê de 
Ética em Pesquisa Humanos da UFJF sob o Parecer $n^{\circ} 386.612$ em 05/09/2013 e CAAE ${ }^{\circ}$ 09148513.1.0000.5147.

\subsection{População e Amostra}

A coleta de dados foi realizada em 07 (sete) academias de ginásticas localizadas em diferentes áreas na cidade de Juiz de Fora, MG. O consentimento dos responsáveis pelos estabelecimentos caracterizou a seleção por conveniência. As mulheres frequentadoras desses estabelecimentos foram a população-alvo desta investigação. Não foi possível realizar o cálculo amostral da população-alvo devido a impossibilidade de identificar o número de estabelecimentos no município junto a diferentes órgãos (CREF e Prefeitura Municipal) que permitisse realizar o levantamento do número de mulheres matriculadas em academias de ginástica na cidade de Juiz de Fora, MG. Dessa forma, a amostra foi selecionada por conveniência, pois as mulheres que estavam presentes nos estabelecimentos nos dias e horários das coletas de dados foram convidadas a participar do estudo. Ao total, 151 mulheres aceitaram participar do estudo e assinaram o Termo de Consentimento Livre e Esclarecido desta pesquisa.

\subsection{Critérios de Inclusão e Não Inclusão}

Foram incluídas na amostra mulheres acima de 21 anos que estavam matriculadas por mais de 03 (três) meses nas academias de ginástica participantes e que tinham disponibilidade para responder o questionário. Não foram incluídas aquelas que possuíam idade superior a 60 anos, estavam em período gestacional ou que tivessem passado por gestação em um intervalo menor que 06 (seis) meses.

\subsection{Instrumento de Coleta de Dados}

Por meio de questionário de autorrelato, coletaram-se dados demográficos (idade, estado civil e escolaridade), massa corporal atual e estatura autorreferidas, massa corporal considerada ideal para si mesma, noções sobre a prática de atividade física e o uso de suplementos alimentares. Para verificar o Índice de Massa Corporal (IMC) das voluntárias, foram utilizados os dados antropométricos autorreferidos, sendo o valor calculado, por meio da fórmula: massa corporal $(\mathrm{kg}) / \mathrm{altura}^{2}(\mathrm{~m})$ (ANJOS, 1992).

A avaliação da insatisfação com a massa corporal foi feita por meio da diferença entre a massa corporal que a pessoa considerava ideal para si mesma e a massa corporal atual auto referida (THOMPSON; VAN DEN BERG, 2002). Para critério de classificação dos dados, foram consideradas satisfeitas aquelas que a diferença foi igual a zero, insatisfeitas desejando aumentar a massa corporal quando o valor da diferença foi positivo e insatisfeitas desejando a redução da massa corporal atual 
quando o valor da diferença foi negativo.

Para verificar o uso de suplementos alimentares, as voluntárias foram questionadas se utilizavam ou não esse tipo de substância, bem como o principal composto presente e objetivo com essa prática.

\subsection{Procedimentos de Coletas de Dados}

Para diversificação da amostra foram feitas pelo menos 03 (três) visitas em diferentes dias e horários, durante o período de funcionamento de cada uma das academias de ginástica. Os dados foram coletados de forma individual e sem comunicação entre as participantes, em salas adequadas e disponibilizadas pelos estabelecimentos. O questionário de autorrelato foi entregue a cada uma das voluntárias de acordo com a disponibilidade, sendo que logo em seguida, as mesmas receberam orientações verbais sobre o preenchimento do instrumento. O questionário foi aplicado em um único momento para cada sujeito da amostra e qualquer dúvida relacionada ao seu preenchimento foi esclarecida pelos pesquisadores. Não houve tempo que delimitasse a manifestação das respostas pelas participantes. Todo o processo de coleta de dados foi realizado pelos pesquisadores no período de outubro a dezembro de 2015.

\subsection{Análise dos Dados}

A idade, IMC, massa corporal atual e estatura autoreferidas são apresentadas por meio de estatística descritiva, sendo utilizadas medidas de tendência central (média) e dispersão (desvio-padrão). A distribuição da (in)satisfação com a massa corporal, o uso de suplementos alimentares, o principal composto dessas substâncias e os objetivos com esta prática, além do número de horas gastas em práticas de atividades físicas são relatadas por meio de frequências relativas e absolutas. Para verificar a associação entre a insatisfação com a massa corporal e o uso de suplementos alimentares, bem como entre a insatisfação com a massa corporal desejando aumentar ou diminuir a mesma e o uso de suplementos alimentares foi utilizado o Teste Qui-Quadrado adotando o nível de significância de $5 \%(p<0,05)$. Todas as análises estatísticas foram realizadas no SPSS versão 21 para Mac.

\section{Resultados}

Fizeram parte deste estudo 151 mulheres com faixa etária entre 21 e 58 anos, que praticavam atividades físicas em 07 academias de ginástica na cidade de Juiz de Fora, MG. A Tabela 1 apresenta dados de caracterização da amostra de acordo com a idade, massa corporal atual (MCA), estatura informada pelo próprio participante e IMC. 
Tabela 1. Características gerais da amostra

\begin{tabular}{cc}
\hline VARIÁVEIS & MEDIA \pm DP \\
\hline Idade & $32,7 \pm 9,9$ anos \\
MCA & $65,5 \pm 11,8 \mathrm{~kg}$ \\
Estatura & $1,63 \pm 0,06 \mathrm{~cm}$ \\
IMC & $24,5 \pm 4,2 \mathrm{~kg} / \mathrm{m}^{2}$ \\
\hline
\end{tabular}

Legenda: DP - desvio padrão; MCA - massa corporal atual; kg - quilograma; $\mathrm{cm}$ - centímetro; IMC Índice de Massa Corporal; $\mathrm{m}$ - metro.

Fonte: Autores

Nesta pesquisa, 61,6\%das mulheres declararam-se solteiras e 29,8\% casadas. Quanto à escolaridade, 27,8\% delas possuíam o Ensino Médio completo, 21,9\% apresentavam Ensino Superior incompleto e, $41,7 \%$ completo.

Por se tratarem academias de ginástica que ofereciam diversas modalidades, observou-se que $65,6 \%$ praticava mais de uma atividade. A distribuição frequencial da amostra no que se refere à modalidade praticada pode ser observada na Tabela 2.

Tabela 2. Distribuição frequencial da amostra no que se refere à modalidade praticada

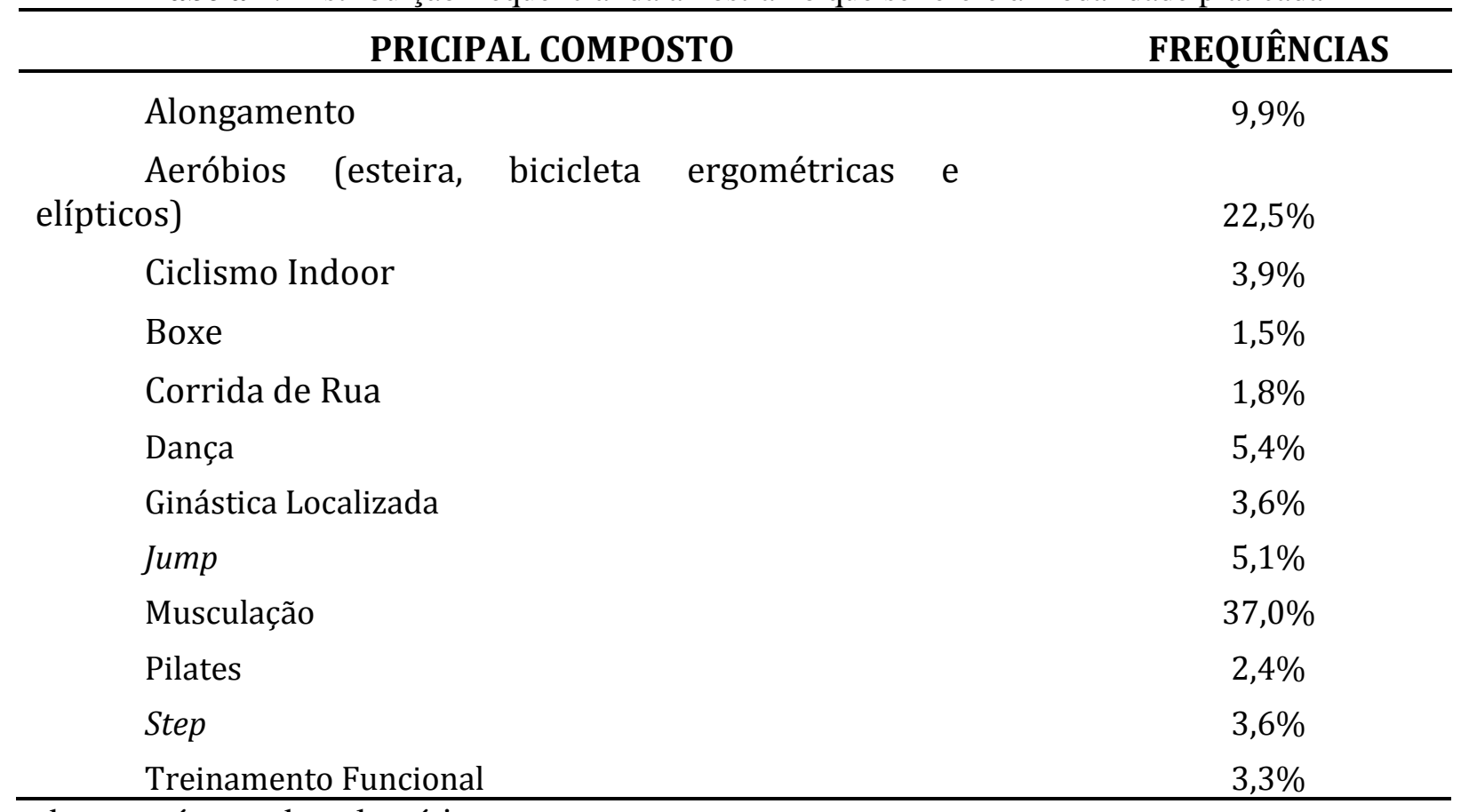

Legenda: $\mathrm{n}=$ número de voluntários.

Fonte: Autores 
A distribuição frequencial das mulheres investigadas quanto ao número de horas dedicadas à prática de atividades físicas diárias em academias de ginástica pode ser observada no Gráfico 1. Destacase que a maior parte delas praticava entre 1 e 2 horas de atividades físicas por dia nas academias de ginástica.

Gráfico 1. Distribuição frequencial das voluntárias de acordo com as horas diárias de atividades físicas praticadas nas academias de ginástica

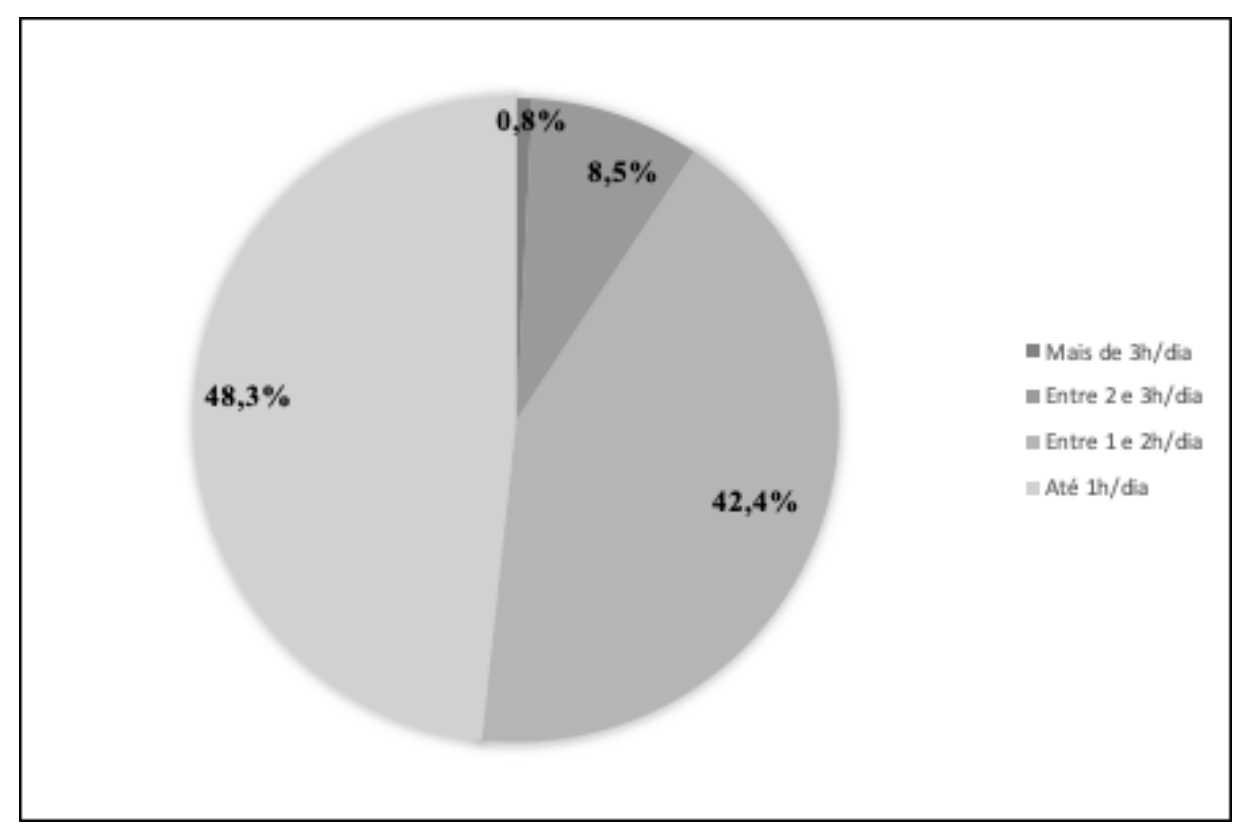

Fonte: Autores

Das 151 mulheres investigadas, observou-se que 91,4\% delas estavam insatisfeitas com a massa corporal, das quais 78,8\% gostariam de reduzir a $\operatorname{MCA}(-7,4 \pm 7,6 \mathrm{~kg})$ e 12,6\% gostariam de aumentá-la $(+3,4 \pm 2,4 \mathrm{~kg})$. Apenas $8,6 \%$ da amostra estava satisfeita com a massa corporal que apresentava no momento da coleta dos dados.

Ao todo, 34,4\%das voluntárias participantes do estudo faziam uso deum ou mais tipos de suplementos alimentares no momento da pesquisa. Na Tabela 3, são apresentadas as frequências relativa e absoluta dos principais compostos dessas substâncias segundo o relato dessas mulheres. 
Tabela 3. Distribuição frequencial das voluntárias de acordo com o principal composto do suplemento alimentar utilizado

\begin{tabular}{lc}
\hline \multicolumn{1}{c}{ PRICIPAL COMPOSTO } & FREQUÊNCIAS \\
\hline Acelerador Metabólico & $30,8 \%$ \\
Aminoácidos & $15,4 \%$ \\
Carboidratos & $11,5 \%$ \\
Proteína & $34,6 \%$ \\
Vitaminas e Minerais & $7,7 \%$ \\
\hline
\end{tabular}

Legenda: $\mathrm{n}$ = número de voluntários.

Fonte: Autores

A distribuição frequencial das voluntárias em função do principal objetivo com o uso dos suplementos alimentares pode ser observado na Tabela 4.

Tabela 4. Distribuição frequencial das voluntárias em função do principal objetivo com o uso de suplementos alimentares

\begin{tabular}{lc}
\multicolumn{1}{c}{ OBJETIVO } & FREQUENCI \\
& AS \\
\hline Aumentar a força & $7,7 \%$ \\
Aumentar a massa muscular & $38,5 \%$ \\
Melhorar a performance & $9,6 \%$ \\
Não sei o motivo & $3,8 \%$ \\
Reduzir a fadiga & $5,8 \%$ \\
Reduzir a massa corporal & $1,9 \%$ \\
Reduzir o percentual de gordura & $19,2 \%$ \\
Repor vitaminas e minerais & $7,7 \%$ \\
Suprir deficiências alimentares & $3,9 \%$ \\
Outros & $1,9 \%$ \\
\hline
\end{tabular}

Legenda: $\mathrm{n}=$ número de voluntários.

Fonte: Autores 
O aumento da massa muscular e a redução da gordura corporal apresentaram maiores frequências, destacando-se como principais objetivos para o uso de suplementos alimentares. Das 52 mulheres frequentadoras de academias de ginástica que faziam uso dessas substâncias no momento do estudo, 73,1\% gostariam de reduzir MCA, 21,2\% desejavam aumentá-la, e apenas, 5,7\% estavam satisfeitas.

Dos 138 indivíduos insatisfeitos com a massa corporal, 35,5\% utilizavam suplementos alimentares, ao passo que, das13 mulheres satisfeitas, apenas 23,1\% possuíam essa prática. Apesar do consumo de suplementos alimentares ser relativamente mais frequente entre as voluntárias insatisfeitas com a massa corporal, não foi possível observar a associação estatisticamente significativa entre essas variáveis $(p=0,544)$.

Das 19 voluntárias insatisfeitas com a massa corporal e desejando aumentá-la, 57,9\% utilizavam suplementos alimentares, assim como, dentre as 119 insatisfeitas com a massa corporal e desejando reduzi-la, 31,9\% apresentavam essa prática. O uso de suplementos alimentares mostrou-se relativamente mais frequente entre as mulheres insatisfeitas com a massa corporal que desejavam aumentá-la, de tal forma que foi possível observar a associação entre essas duas variáveis $(p=0,028)$. Ao todo, 3,5\% da variância no uso de suplementos alimentares pode ser explicado pela insatisfação com a massa corporal, ou seja, pela vontade de aumentar ou reduzir a mesma.

\section{Discussão}

Considerando que tanto homens quanto mulheres insatisfeitos com o próprio corpo tendem se inserir em programas de atividades físicas e a utilizar substâncias para melhorar a aparência física e a performance durante essas práticas (HILDEBRANDT; LAI, 2011; MARINHO; MATTA, 2020), o presente estudo objetivou verificar a associação entre a insatisfação com a massa corporal e o uso de suplementos alimentares em mulheres que frequentam academias de ginástica na cidade de Juiz de Fora, MG.

No presente estudo, quase metade das investigadas dispendia de 1 a 2 horas de atividades físicas por dia nas academias de ginástica, o que representa duração de treino maior quando comparado a estudos anteriores (MARCHIORO; BENETTI, 2015; SOUZA; CENI, 2014) que apontam entre 45 a 60 minutos por dia. Parisotto (2011) sugere que a procura por programas de atividades físicas e o tempo gasto com essas podem estar relacionados à insatisfação corporal dos indivíduos que, na maioria das vezes, objetiva modificar a aparência física do próprio corpo por meio dessas práticas.

Dentre as mulheres entrevistadas, a maior parte mostrou-se insatisfeita com a massa corporal, 
apresentando o desejo de reduzi-la. Esses resultados corroboram estudos (DAMASCENO, et al. 2005; MARINHO; MATTA, 2020), ao apontar que, normalmente, as mulheres tendem a se mostrarem insatisfeitas com a massa corporal, apresentando o desejo de emagrecer.

Ferrari et al. (2012) explicam que a insatisfação das mulheres com o próprio corpo, no geral, é reflexo de não possuírem o estereótipo de corpo magro e com baixo percentual de gordura que estaria em voga na sociedade contemporânea.

Segundo distintos autores (CARVALHO et al., 2013; DAMASCENO et al., 2005; FERRAZ et al., 2015), a busca exacerbada por esse estereótipo pode estimular o engajamento em práticas de atividades físicas e o consumo de suplementos alimentares. Isso parece ocorrer porque a quantidade de gordura corporal, que é um importante fator na manutenção da saúde, nos dias atuais, tornou-se algo de valor estético negativo (ALVES et al. 2017).

$\mathrm{Na}$ atual pesquisa, verificou-se que aproximadamente um terço das mulheres investigadas relatou fazer uso de suplementos alimentares. Diferentes autores (ALVES et al. 2017; LINHARES; LIMA, 2006; HIRSCHBURCH; FISBERG; MOCHIZUKI, 2008; SCHULER; ROCHA, 2018) sugerem que essa expressiva utilização de suplementos alimentares em mulheres parece ser reflexo do papel que essas substâncias assumem como uma estratégia que possibilitaria modificar a aparência física do próprio corpo, em prol de alcançar padrões corporais socialmente construídos.

Segundo as voluntárias que declararam consumir suplementos alimentares, os compostos mais frequentes nessas substâncias eram proteínas e aceleradores metabólicos, concordando com achados de estudos anteriores (FERRAZ et al., 2015; FRADE et al., 2016; SCHULER; ROCHA, 2018). Consequentemente, o uso de suplementos alimentares se justificou devido as mesmas visualizarem a necessidade de promover modificações na composição corporal, principalmente no sentido de aumentar massa muscular e reduzir gordura corporal, corroborando investigações existentes na literatura (ROTH; BOSCAINI, 2014; SCHULER; ROCHA, 2018).

Fayer et al. (2013) salientam que apesar do consumo de suplementos alimentares apresentar-se como um comportamento corriqueiro entre os frequentadores das academias de ginástica, a utilização dessas substâncias sem orientação nutricional parece ser perigosa, pois pode causar diversos efeitos prejudiciais a seus usuários.

Os resultados aqui apresentados corroboram investigações anteriores (PASSAGLIA et al., 2015; SILVA; FERREIRA JUNIOR; NEVES, 2012) ao apontar que o consumo de suplementos alimentares pode ser observado em mulheres insatisfeitas com a massa corporal. Entretanto, neste estudo não foi possível observar associação entre a insatisfação com a massa corporal e o uso de suplementos alimentares em mulheres praticantes de atividades físicas. 
De maneira a contribuir para literatura recente, observamos que o uso de suplementos alimentares pode ser verificado tanto em mulheres que desejam reduzir a massa corporal, quanto aqueles que almejam aumentá-la. Nesse sentido, foi possível detectar associação entre a insatisfação com a massa corporal no sentido de aumentá-la e o uso de suplementos alimentares. Esse achado possivelmente pode ser explicado, devido ao fato de que os indivíduos que consomem essas substâncias, normalmente, justificam essa prática devido à intenção de aumentar a massa muscular (FRADE et al, 2016; SCHULER; ROCHA, 2018), o que também pode implicar no ganho de massa corporal.

A primeira limitação deste estudo refere-se à utilização de uma amostra não probabilística de mulheres praticantes de atividades físicas em academias de ginástica da cidade de Juiz de Fora, MG, o que inviabiliza a generalização dos resultados para todo o município. Essa dificuldade em obter o número de academias de ginástica em Juiz de Fora, MG, que possibilite o levantamento de populações-alvos existentes nesses estabelecimentos, já foi apontado por Rosa Junior et al. (2015). Outra limitação diz respeito á utilização de dados antropométricos auto referidos (massa corporal e estatura) para cálculo de IMC, contudo pesquisas existentes na literatura (FONSECA et al, 2004; OLIVEIRA et al., 2008) atestam a utilização desses dados para essa finalidade.

Curi e Bueno Júnior (2011) ressaltam a importância de considerar a influência de distintos contextos socioculturais sobre a insatisfação com o próprio corpo, como um reflexo de demandas presentes na sociedade contemporânea que estão relacionadas à aparência física dos indivíduos. Dessa forma, as informações apresentadas aqui servem de subsídios para que profissionais de Educação Física e Nutricionistas realizem intervenções junto a mulheres frequentadoras de academias de ginástica.

Por fim, sugere-se que novos estudos abrangendo mulheres praticantes de atividades físicas em academias de ginástica e aspectos da imagem corporal sejam desenvolvidos, promovendo melhor conhecimento das interrelações entre os mesmos.

\section{Considerações Finais}

No presente estudo, nota-se que boa parte das mulheres praticantes de atividades físicas em academias de ginástica da cidade de Juiz de Fora, MG que foram investigadas encontravam-se insatisfeitas com a massa corporal. Dentre essas voluntárias observou-se tanto o desejo de reduzir a massa corporal, quanto a vontade de aumentá-la.

Quanto ao uso de suplementos alimentares, aproximadamente um terço das voluntárias investigadas consumiam algum tipo de substância. No que se refere aos objetivos com essa prática, evidenciou-se a vontade de aumentar a massa muscular e a reduzir a gordura corporal, dentre outros. 
Contudo, apesar dessas constatações, não foi possível observar associação direta entre a insatisfação com a massa corporal e o uso de suplementos alimentares. De maneira mais específica, o consumo dessas substâncias parece ser mais frequente entre indivíduos insatisfeitos com a massa corporal que apresentam o desejo de aumentá-la, de maneira que possibilitou observar associação entre essas duas variáveis.

Pode-se conjecturar que o engajamento em práticas de atividades físicas e o consumo de suplementos alimentares com objetivo de promover modificações no próprio corpo parece não ser suficiente para proporcionar a satisfação da mulher contemporânea em relação a aspectos da imagem corporal, em especial, a massa corporal. Uma possível explicação, seria a pressão exercida por fatores socioculturais (pais, amigos e mídias) sobre os indivíduos, no sentido de estimular uma contínua busca pela adequação do próprio corpo baseada em padrões corporais socialmente idealizados.

Entretanto, espera-se que profissionais das áreas de Educação Física e Nutrição estejam atentos para realizar intervenções junto aos seus clientes, no sentido de estimular práticas saudáveis de atividades físicas e alimentação, respectivamente. Atuações estas que vislumbrem ressaltar a importância das ações, considerando os objetivos pessoais, características individuais, potencialidades do próprio corpo e autoaceitação corporal, indo para além da aparência física.

\section{Referências}

ADAMI, F. et al. Insatisfação corporal e atividade física em adolescentes da região continental de Florianópolis. Psicologia: Teoria e Pesquisa, Brasília, v.24, n. 2, p.143-149, abr./ jun. 2008.

ALVARENGA, S.M. et al. Insatisfação com a imagem corporal em universitárias brasileiras. Jornal Brasileiro de Psiquiatria, São Paulo, v. 59, n. 1, p. 44-51, mar. 2010.

ALVES et al. Insatisfação com a imagem corporal e fatores associados em universitários. Revista Interdisciplinar de Promoção da Saúde. v. 18. n. 3. p.1-6. 2017.

AMARAL, A. C. S.; CARVAlHO, P. H. B.; FERREIRA, M. E. C. A cultura do corpo perfeito: a influência sociocultural na Imagem Corporal. In: FERREIRA, M. E. C.; CASTRO, M. R.; MORGADO, F. F. R. (Org.). Imagem Corporal: reflexões, diretrizes e práticas de pesquisa. Juiz de Fora: Editora da UFJF, 2014. p. 173-186.

ANJOS, L.A. Índice de massa corporal (massa corporal. estatura2) como indicador do estado nutricional de adultos: revisão da literatura. Revista de Saúde Pública, São Paulo, v.26, n.6, p.431-436, dez. 1992.

CARVALHO, B. H. P. et al. Checagem corporal, atitude alimentar inadequada e insatisfação com a imagem corporal de jovens universitários. Jornal Brasileiro de Psiquiatria, Rio de Janeiro, v. 62, n. 2, p. 108-114, abr. 2013. 
CARVALHO, B. H. P.; CASTRO, R. M.; FERREIRA, C. E. M. Imagem Corporal e Vida Adulta. . In: FERREIRA, M. E. C.; CASTRO, M. R.; MORGADO, F. F. Imagem Corporal, reflexões, diretrizes e práticas de pesquisa. Juiz de Fora: editora UFJF, 2014. p. 87-114.

CASH, T. F.; SMOLAK, L. Understanding Body Image: Historical and contemporary perspectives. In: CASH; T.F.; SMOLAK, L. (Ed.). Body Image: A Handbook of Science, Practice, and Prevention. $2^{\text {nd }}$ ed. New York: The Guilford Press, 2011. p. 3-11.

CFN. Resolução $\mathbf{n}^{\mathbf{0}}$ 380/2005, de 28 de dezembro de 2005. Dispõe sobre as áreas de atuação do Nutricionista e suas atribuições, estabelece parâmetros numéricos de referência, por área de atuação, e dá outras providências. Resolução $\mathrm{n}^{\circ}$ 380, de 28 de dezembro de 2005. Disponível em: <http://www.cfn.org.br/novosite/pdf/res/2005/res380.pdf> . Acesso em: 06 jul. 2015.

CURI, G. I.; BUENO JÚNIOR, C. R. Prevalência de distorção da imagem corporal em mulheres eutróficas com sobrepeso e obesas frequentadoras de academias de ginástica. Revista Brasileira de Obesidade, Nutrição e Emagrecimento, São Paulo, v. 5, n. 23, p. 17-23, jan./fev. 2011.

DAMASCENO, O. V. et al. Tipo físico ideal e satisfação com a imagem corporal de praticantes de caminhada. Revista Brasileira de Medicina do Esporte, São Paulo, v. 11, n. 3, p. 181-186, mai.jun. 2005.

FAYER, A.P.T et al. Consumo de suplementos nutricionais por frequentadores de academias da cidade de Porto Alegre. Revista Brasileira de Ciência do Esporte, Florianópolis, v. 35, n. 1, p. 27-37, jan./mar. 2013.

FERRAZ, B. S. et al. Consumo de suplementos alimentares por praticantes de atividades físicas e academias de ginástica: um artigo de revisão. Journal of Amazon Health Science, Rio Branco, v. 1, n. 2, p.24-43, 2015.

FERRARI, E.P. et al. Insatisfação com a imagem corporal e relação com o nível de atividade física e estado nutricional em universitários. Revista Motricidade, Ribeira de Pena, vol. 8, n. 3, p. 52-58, ago. 2012.

FONSECA, M. J. M. et al. Validade de peso e estatura informados e índice de massa corporal: estudo pró-saúde. Revista de Saúde Pública, São Paulo, v.38, n.3, p. 392-398, jun. 2004.

FRADE, R. E. T. et al. Avaliação do consumo de suplementos nutricionais por frequentadores de uma academia de ginástica de São Paulo - SP. Revista Brasileira de Nutrição Esportiva, São Paulo, v. 10, n. 55, p.50-58, jan./fev., 2016.

GROGAN, S. Body Image: understanding body dissatisfaction in men, women, and children. $2^{\text {nd }}$ ed. New York: Psychology Press, 2008.

HIRSCHBRUCH, M.D.; FISBERG, M.; MOCHIZUKI, L. Consumo de suplementos por jovens frequentadores de academias de ginástica em São Paulo. Revista Brasileira de Medicina do Esporte, São Paulo,vol. 14, n. 6, p. 539-543, nov./dez. 2008. 
HILDEBRANDT, T.; LAI, J. Body Image and Appearance - and Performance - Enhancing Drug Use. In: CASH, T. F.; SMOLAK, L. Body image: A handbook of science, practice, and prevention. $2^{\text {nd }} \mathrm{Ed}$. New York: The Guilford Press, 2011. p. 3-11.

IRIART, B. A. J.; CHAVES, C. J.; ORLEANS, G. R. Culto ao corpo e uso de anabolizantes entre praticantes de musculação. Cadernos Saúde Pública, Rio de Janeiro, v.25, n. 4, p. 773-782, abr. 2009.

KAKESHITA, S. I.; ALMEIDA, S. S. Relação entre índice de massa corporal e a percepção da autoimagem em universitários. Revista Saúde Pública, Ribeirão Preto, v. 40, n. 3, p. 497-504, fev. 2006.

LINHARES, T.C.; LIMA, R,M. Prevalência do uso de suplementos alimentares por praticantes de musculação nas academias de Campos dos Goytacazes/RJ, Brasil. VÉRTICES, Campos dos Goytacazes, v. 8, n. 1, p. 101-122, jan./dez. 2006.

MARCHIORO, E. M.; BENETTI, F. Consumo de suplementos nutricionais por praticantes de musculação em academias do município de Tenente Portela - RS. Revista Brasileira de Nutrição Esportiva, São Paulo, v. 9, n. 49, p. 40-52, jan./fev. 2015.

MARINHO, S. M. S. A.; MATTA, S. S. Physical Activity and Body Image Perception in Women: a systematic review. Journal of Sports and Physical Education, v.7, n.2, p.40-44, mar-apr.2020.

MOREIRA, N. M.; NAVARRO, A. C.; NAVARRO, F. et al. Consumo De Suplementos Alimentares Em Academias De Cachoeiro De Itapemirim-Es. Revista Brasileira de Nutrição Esportiva, São Paulo. v. 8. n. 48. p.363-372,nov./dez. 2014.

OLIVEIRA, A. G. P. et al. Diferença entre peso e estatura auto-referidos e aferidos para o cálculo de índice de massa corporal e a sua relação com a imagem corporal de mulheres de academia de ginástica. HU Revista, Juiz de Fora, v. 34, n. 3, p. 179-183, jul./set. 2008.

PARISOTTO, C.D. Relação entre índice de massa corporal e a insatisfação com a autoimagem em mulheres praticantes de musculação.2011. 46f. TCC (Graduação) - Curso de Educação Física: Licenciatura, Escola de Educação Física, Universidade Federal do Rio Grande do Sul, Porto Alegre, 2011.

PASSAGLIA, A. P. et al. Análise do perfil de usuários de academias em Alfenas-MG. Revista Brasileira de Nutrição Esportiva, São Paulo, v. 9, n. 53, p. 471-479, set./out. 2015.

PEREIRA, F.E. et al. Relação entre diferentes indicadores antropométricos e a percepção da imagem corporal em idosas ativas. Revista de Psiquiatria Clínica, Rio Grande do Sul, v. 36, n. 2, p. 54-59, jan. 2009.

ROSA JUNIOR, C. R. R. et al. Correlações entre insatisfação corporal, estado nutricional e comportamentos de checagem do corpo em profissionais de Educação Física atuantes em academias de ginástica. HU Revista, Juiz de Fora, v.41, n.1 e 2, p. 65-70, jan./jun. 2015.

ROTH, K. D. T.; BOSCAINI, C. Estado nutricional, exercícios físico e uso de suplementos em indivíduos de uma academia da Serra Gaúcha - RS. Revista Brasileira de Nutrição Esportiva, São Paulo, v. 8, n. 46, p. 217-227, jul./ago. 2014. 
RUSSO, L.; TIRAPEGUI, J. Body image dissatisfaction among gym-goers in Brazil. Revista Brasileira de Medicina do Esporte, São Paulo, vol. 24, n. 2, p. 162-166, mar./abr. 2008.

SCHULE, A. .C; ROCHA, R. E. R. Fatores associados à utilização de suplementos alimentares por universitários. Revista Brasileira de Nutrição Esportiva, São Paulo, v.12, n.73, p.590-597, set./out. 2018.

SILVA, R. K.; FERREIRA JUNIOR, D. A. F.; NEVES, A. S. Consumo de ergogênicos nutricionais por praticantes de musculação de diversas academia de ginástica de Resende-RJ. Revista Brasileira de Nutrição Esportiva, São Paulo, v. 6, n. 36, p. 470-476, nov./dez. 2012.

SOUZA, R.; CENI, G.C. Uso de suplementos alimentares e auto percepção corporal e praticantes de musculação em academias de Palmeira Das Missões - RS. Revista Brasileira de Nutrição Esportiva, São Paulo, v. 8, n. 43, p.20-29, jan./fev. 2014.

THOMAS, J.R.; NELSON, J.K.; SILVERMAN, S.J. Métodos de pesquisa em atividade física. Traduzido por: Ricardo Demétrio de Souza Petersen. 6 Edição. Porto Alegre: Artmed, 2012.

THOMPSON, J. K; VAN DEN BERG, P. Measuring body image attitudes among adolescents and adults. In: CASH, T. F.; PRUZINSKY, T. (Org.). Body image: a handbook of theory, research, \& clinical practice. New York: Guilford, 2002. p. 142-154.

THORNSBORROW, T. et al. Muscles and Media: a natural experiment across cultures in men's body image. Frontiers in Psychology, Lausanne, v. 11, n. 11, p.1-16, apr. 2020.

\section{Como citar este artigo}

ROSA JUNIOR, C. R. R.; VIANA, J. F.; SOUZA, M. R. V.; FERREIRA, M. E. C. Insatisfação com a massa corporal e uso de suplementos alimentares em mulheres que frequentam academias de ginástica em Juiz de Fora - MG. Revista Kinesis, Santa Maria, v.38, p.01-15, 2020. 